\title{
How good are doctors at estimating children's weight?
}

\author{
A Greig, J Ryan, E Glucksman
}

\section{Department of Accident and Emergency Medicine, King's College Hospital, Denmark Hill, London SE5 9RS, United Kingdom A Greig J Ryan \\ E Glucksman \\ Correspondence to: Dr Aina V H Greig, Senior House Officer Department of ENT Surgery, St Thomas' Hospital, Lambeth Palace Rd, London SE1 7EH.}

Accepted for publication 13 November 1996

\begin{abstract}
Objective-(1) To see whether children are weighed before drugs are prescribed in an accident and emergency (A\&E) department; (2) to assess how safe it is for doctors to guess children's weight if they prescribe "by eye".

Methods-An audit of 100 sets of notes was performed to see if children were weighed before drugs were prescribed. A\&E senior house officers were asked to estimate the weight of 75 children aged 12 years and under.
\end{abstract}

Results-Children were weighed before prescribing in only two out of 100 cases. The mean difference between the actual and estimated weights was -0.21 (not significantly different from zero: $P=0.40$ ); $95 \%$ of the estimates were within two standard deviations of the mean difference. The percentage difference between the actual and estimated weight varied between overestimates of $300 \%$ and underestimates of nearly $100 \%$.

Conclusions-The average guess of doctors as a group was approximately correct. However, there was a wide range of estimates for individuals. If the child's weight is guessed, the doctor could risk under- or overprescribing analgesia, sedation, or intravenous fluids. Given the

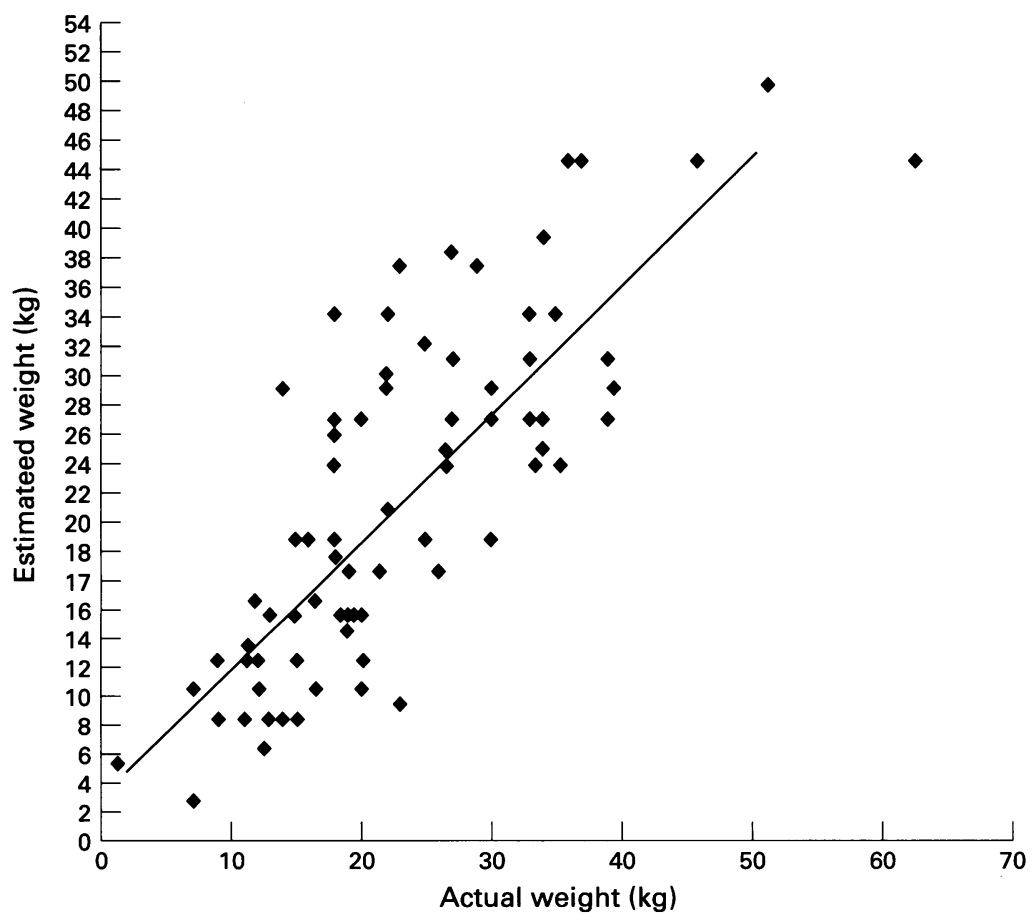

Figure 1 Regression line drawn on scatter diagram relating actual and estimated weight $(\mathrm{kg})$ of 75 children. wide range of estimates, actual weights are required for accurate prescribing. Prescribing on an age basis may be acceptable for drugs such as paracetamol or amoxycillin, but it is imperative to prescribe on a $\mathrm{mg} / \mathrm{kg}$ basis for opiates, sedatives, and intravenous fluids because of the large variation in weight that can occur for a single age. $(\mathcal{F}$ Accid Emerg Med 1997;14:101-103)

Keywords: weight estimation; drugs; children.

Weighing children may be standard practice in paediatric accident and emergency (A\&E) departments, but our experience suggests that this is not routine practice in general $A \& E$ departments. A survey in our $A \& E$ department confirmed that children were often not weighed before drugs or fluids were prescribed. Concern about dosage inaccuracies, ${ }^{1}$ led to a formal review of current practice in our department with regard to weighing of children, and in particular the use of the children's actual weight when drugs or fluids were prescribed.

\section{Methods}

$\mathrm{A} \& \mathrm{E}$ notes of 100 paediatric attendances where drugs or intravenous fluids were prescribed were reviewed. Note was made of age and whether the child's weight was documented.

Eight $A \& E$ senior house officers were asked to estimate the weights of 75 children aged 12 years and under who visited the department. The doctors were told the age, in years and months, of the child before guessing the weight. The actual weights of these children were recorded, but not revealed to the doctors involved in the study. None of these doctors had previously had a paediatric appointment.

A paired $t$ test was used to see if the mean difference was significantly different from zero.

The regression coefficient was calculated and a regression line was drawn on a scatter diagram relating actual and estimated weight to see if there was a positive correlation between changes in the actual weight and the accuracy of the estimation.

The percentage difference between the estimated and actual weights was calculated and these values were plotted against the actual weights, to see if the points fell within an acceptably small range.

An analysis was made of how good individual doctors were at estimating children's weight. The mean difference between the 


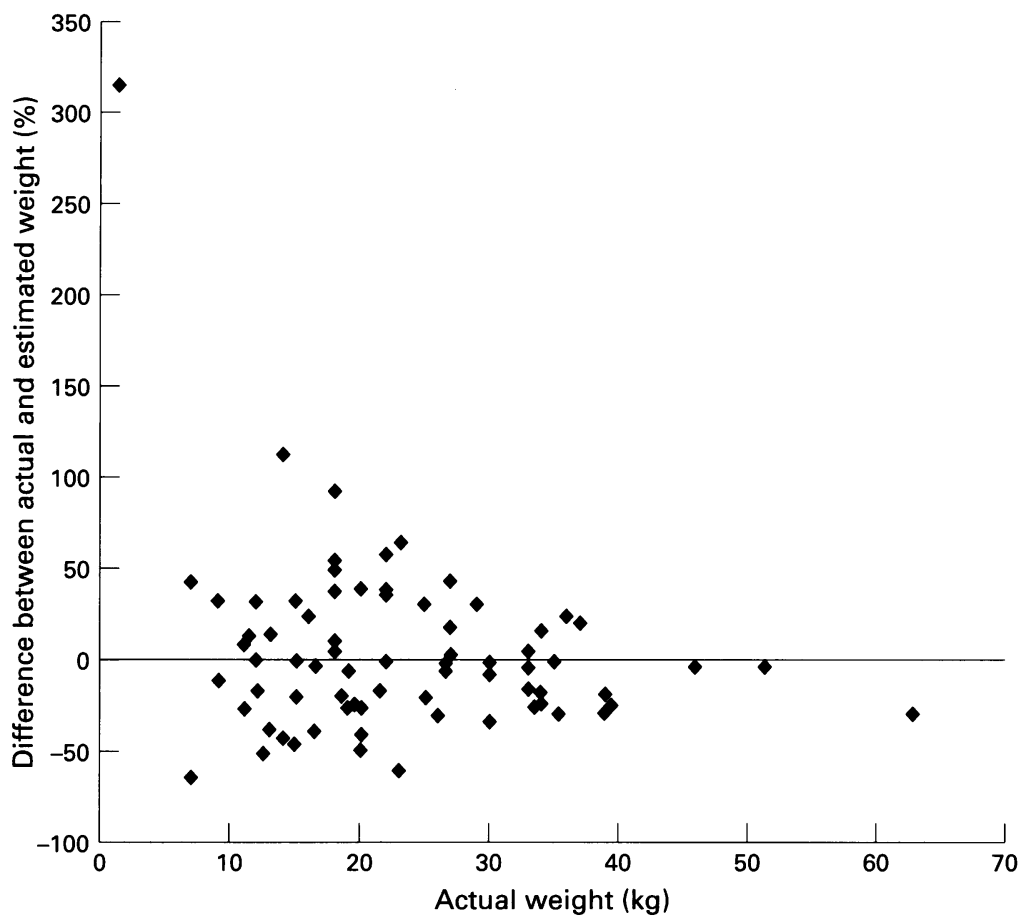

Figure 2 Actual weight $v$ percentage difference between actual and estimated weight.

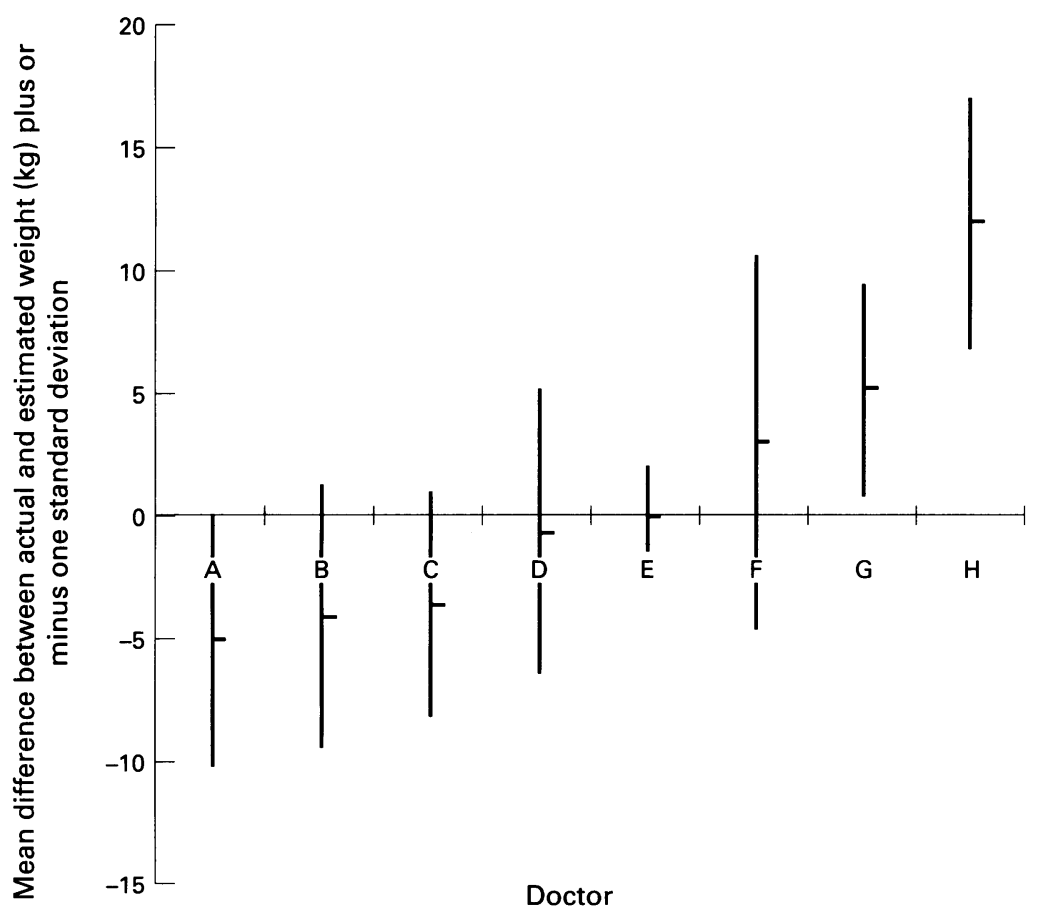

Figure 3 How good are individual doctors at guessing children's weight?

actual and estimated weights, for each of the eight doctors participating in the study, was calculated with the standard deviation and the result plotted.

\section{Results}

The patient's weight was recorded in only two out of 100 sets of notes where drugs or fluids were prescribed. These two patients were prescribed sodium valproate and glycerine suppositories. Drugs given without children being weighed included: antibiotics, analgesics, antiemetics, sedatives, bronchodilators, antihistamines, and emetics. One child was prescribed intravenous fluids without being weighed. This was in an emergency situation where the child had collapsed and fitted after severe diarrhoea and dehydration.

The mean difference between the actual and estimated weights was -0.21 . The mean difference was not significantly different from zero: $P=0.40$. The standard deviation of the mean difference was $7.14 ; 95 \%$ of the estimates were within two standard deviations of the mean difference. The regression coefficient was 0.85 . Therefore the ability of doctors to estimate weight correlated with the age and weight of the child (fig 1). The lower the weight of the child, the larger the error in weight estimation (fig 2).

The mean difference between the actual and estimated weights, for each of the eight doctors participating in the study, is shown with standard deviation in fig 3. The percentage difference between the actual and estimated weight varied between overestimates of $300 \%$ and underestimates of nearly $100 \%$. The child whose weight was overestimated by more than $300 \%$ was one month old and the actual weight $(1.2 \mathrm{~kg})$ was less than the normal range for that age. Another child whose weight was overestimated by more than $100 \%$ was four years and two months old and the actual weight $(14 \mathrm{~kg})$ was within the normal range.

\section{Discussion}

Are doctors good enough at estimating children's weights so that they can safely prescribe "by eye"? For the whole group of doctors the mean difference between actual and estimated weight was not significantly different from zero. Therefore in general the average guess of the whole group was approximately correct. However, this is dealing with a group and not individuals, and we are interested in the performance of individuals.

Considering individuals, the range of estimates was wide. This is because the estimates of eight different doctors are superimposed. On analysing the estimates of individual doctors we found that there was much variability between doctors, but little variability for one individual - that is, individual doctors tended to be consistent over- or underestimators of weight. Therefore, although individual doctors appear consistent and to have specific patterns of behaviour, the patterns are very varied and interobserver error is large enough for it to be mandatory that all children are weighed to minimise risk. The range of weights for the six year olds who were weighed in this study was 19.5 to $30 \mathrm{~kg}$. This is a large variation, suggesting that it is not satisfactory to prescribe by age alone. A child may end up significantly underor over-dosed.

Children aged from 0 to one year were not routinely seen by casualty officers in our department unless they were involved in an accident. Therefore the wide range of estimates for children of this group was partly due to unfamiliarity of the doctors carrying out the assessment in children of that age, and this is all the more reason to weigh such children before prescribing. 
CONCLUSIONS

Paediatric patients are not usually weighed and doctors prescribe either on an age basis, or by estimating children's weights. The percentage difference between the actual and estimated weight varied between overestimates of $300 \%$ to underestimates of nearly $100 \%$. This range is too wide for it to be clinically acceptable to guess the weights of children when prescribing on a $\mathrm{mg} / \mathrm{kg}$ basis. Actual weights are essential for accurate prescribing.

Prescribing by age alone may be acceptable for drugs like paracetamol or amoxycillin, but it is best practice to prescribe on a $\mathrm{mg} / \mathrm{kg}$ basis for opiates, sedatives, and intravenous fluids. In emergency situations when a child is too ill or too injured to be weighed, it is possible to use a Broselow tape, ${ }^{2}$ Oakley's paediatric resuscitation chart, ${ }^{3}$ or the accepted emergency formula for estimating a child's weight [weight in $\mathrm{kg}=$ 2 (age +4$)]$ as recommended by APLS.

Obtaining accurate weights would mean a small change in current practice, which would cost little and would only involve a small increase in time spent by nurses in their assessment of patients and by doctors when working out appropriate doses. This small change could have a significant impact in preventing critical incidents with paediatric drug and fluid prescribing in the $A \& E$ department.

Thanks to Dr M Message for statistical help and advice.

1 Waters MR. Paediatric resuscitation: dosage inaccuracies may be dangerous. BMJ 1993;306:1612-3.

2 Hughes G, Spoudeas H, Kovar IZ, Millington HT. Tape measure to aid prescription in paediatric resuscitation. Arch Emerg Med 1990;7:21-7.

3 Oakley PA. Inaccuracy and delay in decision making in paediatric resuscitation, and a proposed reference chart to reduce error. BMJ 1988;297:817-9.

\section{EMERGENCY CASEBOOK}

\section{Nocturnal hypoglycaemia: a cause of bilateral anterior shoulder dislocation}

A 40 year old Caucasian man who was an insulin dependent diabetic presented to the A\&E department. His wife reported that 11 days before this attendance he had been unusually restless while asleep in bed. The next morning he awoke with stiff painful shoulders which failed to resolve over the next week. Clinical examination showed typical loss of normal contour and reduced movement of both shoulders. Axillary nerve function was intact, but there were bilateral paraesthesiae in the C6/7 distribution. Blood glucose concentration was $13.9 \mathrm{mmol} /$ litre. $X$ ray revealed anterior dislocations of both shoulders (figure). Attempted reduction in the $A \& E$ department under sedation failed; both shoulders were reduced under general anaesthesia using the Hippocratic method and his arms supported by collar and cuffs with a chest strap. Axillary nerve function remained intact and the paraesthesiae resolved within a few weeks. Shoulder movements returned to normal with no apparent instability.

Nocturnal hypoglycaemia is said to be more prevalent if bedtime blood glucose concentration is below 7 $\mathrm{mmol} / \mathrm{litre}$ in adults. The classical presentations of nocturnal hypoglycaemia are night sweats or nightmares and morning headaches. Reporting of hypoglycaemia by diabetic patients is known to be unreliable; this is thought to be because hypoglycaemia impairs cerebral function. Thus patients' partners may be more reliable historians. Some insulin dependent diabetics have an impaired ability to prevent hypoglycaemia, developing neuroglycopenia leading to
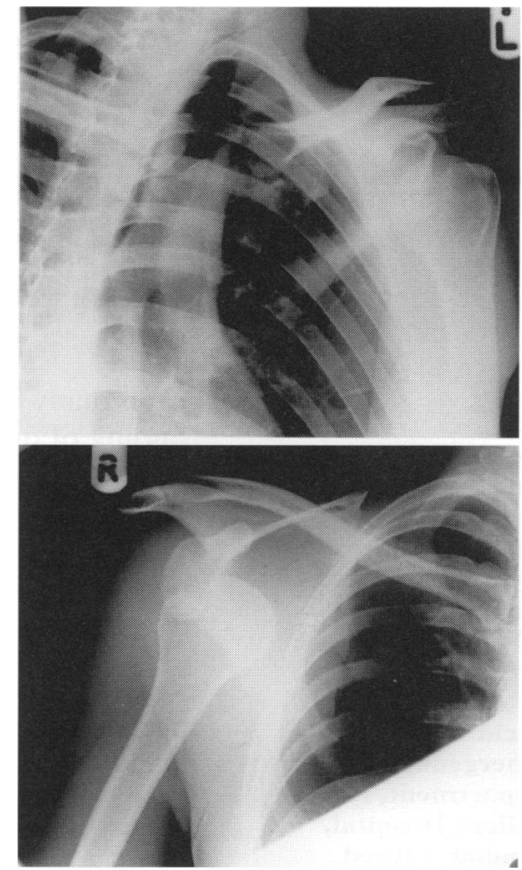
convulsions at higher plasma glucose levels than would be expected. This explains how convulsions can follow only a modest fall in blood glucose.

A J ANDrade, C MANN, M L Grover, Department of Orthopaedics, Queen Alexandra Hospital, Cosham, Portsmouth PO6 $3 L Y$ 